\title{
Hybrid photon-plasmon nanowire lasers
}

Xiaoqin $\mathrm{Wu}^{1 \dagger}$, Yao Xiao ${ }^{1 \dagger}$, Chao Meng ${ }^{l}$, Xining Zhang ${ }^{l}$, Shaoliang Yu ${ }^{l}$, Yipei Wang ${ }^{l}$, Chuanxi Yang ${ }^{1}$, Xin Guo ${ }^{1}$, C. Z. Ning ${ }^{2}$, Limin Tong ${ }^{1 *}$

${ }^{1}$ State Key Laboratory of Modern Optical Instrumentation, Department of Optical Engineering, Zhejiang University, Hangzhou 310027, China

${ }^{2}$ School of Electrical, Computer and Energy Engineering, Arizona State University, Tempe, AZ 85287, USA

${ }^{\dagger}$ These authors contribute equally to this paper. $\quad *$ Corresponds: phytong@zju.edu.cn 


\section{Supporting Information}

\section{Selected-area spectral measurement system}

The spatial-resolved optical characterization and spectral measurement of end-facet emission of both CdSe and Ag NWs are carried out under the selected-area spectral measurement system schematically illustrated in Fig. S1. Based on an optical microscopy, laser pulses from a 532-nm-wavelength laser ( $2 \mathrm{kHz}$ repetition rate, $5 \mathrm{~ns}$ pulse width) are focused into a $25-\mu \mathrm{m}$-diameter spot (via a $100 \times$ objective) for NW excitation. The supporting substrate here is a $\mathrm{MgF}_{2}$ crystal. The emission from the end-facet of a NW is collected with the same objective, and directed to a spectrometer and a CCD camera for spectral measurement and imaging, respectively.

A 532-nm notch filter is inserted to block the pumping light. A two-dimensionally movable pinhole is employed on the imaging plane to selectively collect light from the NW end-facet with a spatial resolution of about $3 \mu \mathrm{m}$.

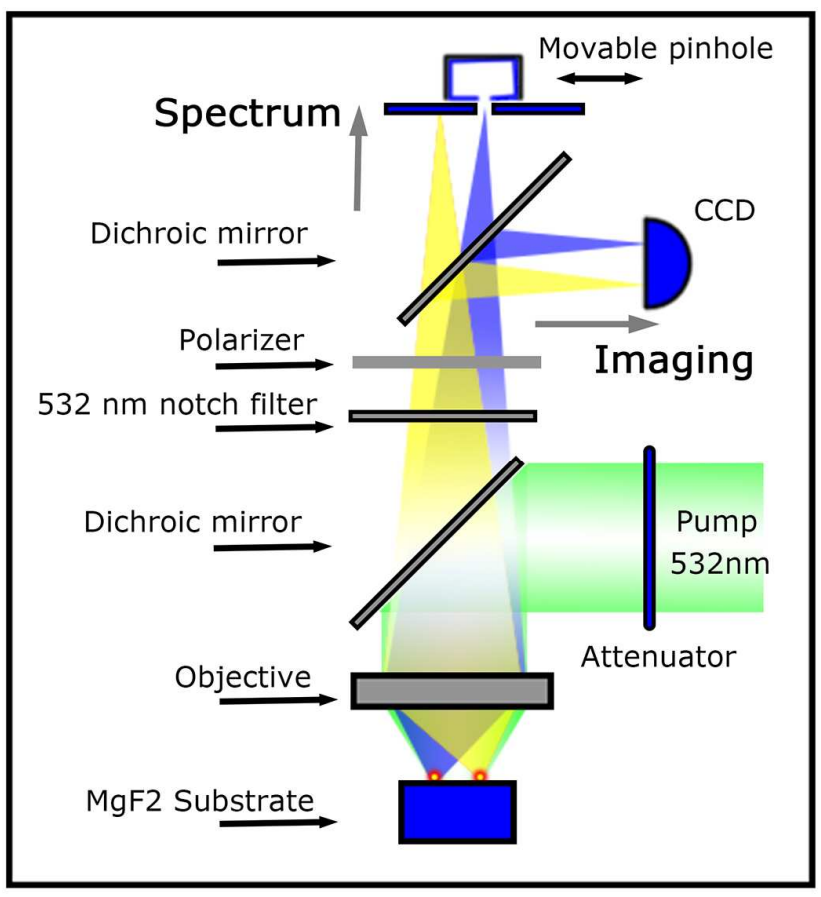

Figure S1. Schematic diagram of selected-area spectral measurement system for spatial-resolved optical characterization. 


\section{End-facet reflectivity of Ag NWs}

To investigate the end-facet reflectivity of Ag NW, we use a prism coupling configuration (Figure S2.a) to excite propagating SPP in the NW with a halogen lamp. The scattered light from the end-facet (Figure S2.inset) is collected by a microscope objective $(100 \times, \mathrm{NA}=0.70)$. Figure S2.b shows the spectrum taken from the right end-facet of a 100-nm-diameter 2.5- $\mu \mathrm{m}$-length $\mathrm{Ag}$ NW, with evident spectral modulation by the NW F-P cavity. The relative modulation depth (see Figure S2.b) is related to the propagation loss $A$ and end-facet reflectivity $R$ by ${ }^{1}$

$$
\frac{\Delta I}{I_{\min }}=\frac{4 R A}{(1-R A)^{2}}
$$

The propagation loss $A$ is defined by $A=e^{-L / l}$, where $L$ is the NW length and $l$ is the SPP propagation length. By assuming $l$ to be $10 \mu \mathrm{m}$ (equivalently $0.43 \mathrm{~dB} / \mu \mathrm{m}$ [Ref.2]), we obtain end-facet reflectivity of about $25 \%$, which is in good agreement with previously reported experimental results ${ }^{1}$

a
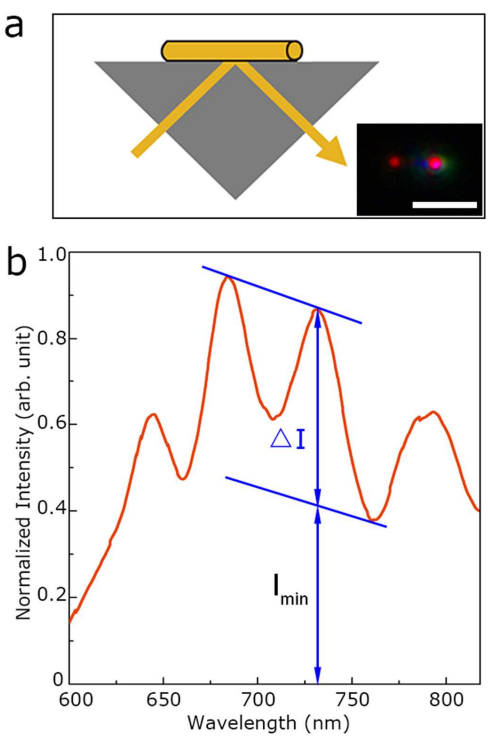

Figure S2. Scattered light spectra for a 100-nm-diameter 2.5- $\mu$ m-length Ag NW. a, Configuration of optical excitation. Inset is CCD image of the excited Ag NW. b, Scattered light spectrum from the right end-facet of the Ag NW. 


\section{Absorption-induced propagation loss of a CdSe NW}

To estimate the absorption-induced propagation loss of a single CdSe NW, we focus a 532 $\mathrm{nm}$ light onto a 390-nm-diameter 500- $\mu \mathrm{m}$-length CdSe NW with a $25-\mu \mathrm{m}$-diameter excitation spot, and scan the spot along the length of the NW (Figure S3 a-g) while collecting the propagation-length-dependent PL from its end-facet with a spectrometer (see Figure S1).At the wavelength around the PL peak (here we use 710,720 and $730 \mathrm{~nm}$, respectively), the output intensity decreases exponentially with the propagation length along the NW (i.e., obeys the Lambert- Beer law), with estimated absorption coefficients of $3590 \mathrm{~cm}^{-1}(710 \mathrm{~nm}), 3025 \mathrm{~cm}^{-1}$ (720 $\mathrm{nm})$ and $576 \mathrm{~cm}^{-1}(730 \mathrm{~nm})$, respectively.

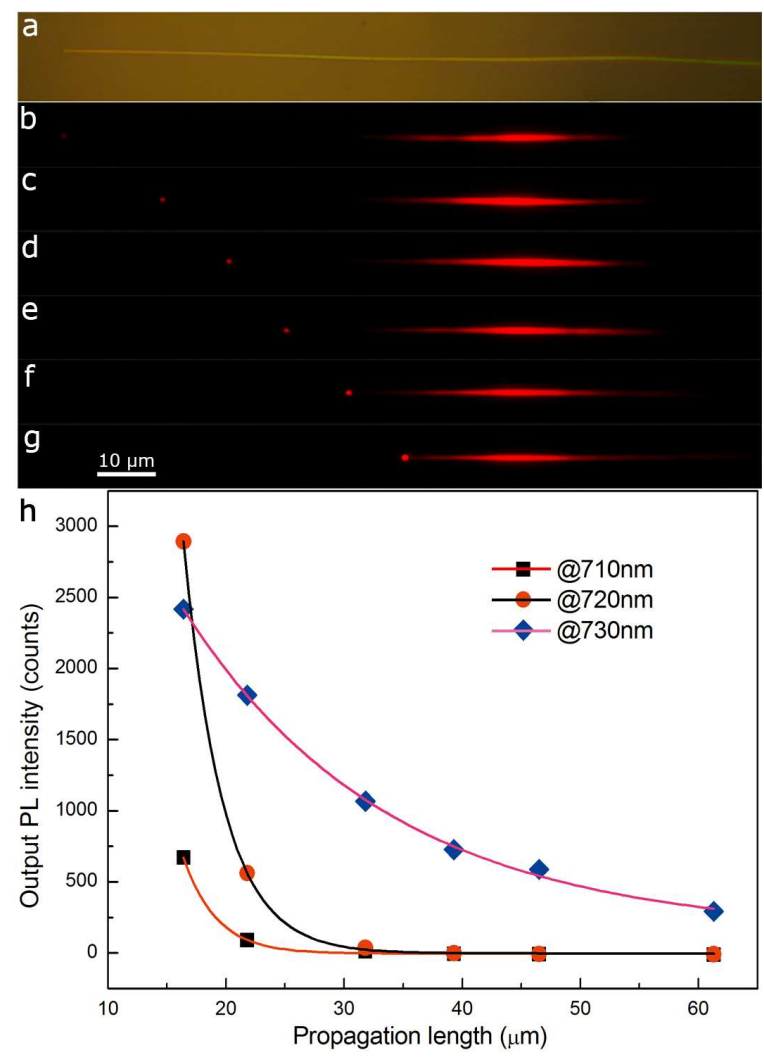

Figure S3. Measurement of absorption-induced propagation loss of a CdSe NW. a, Bright-field optical microscope image of a 390-nm-diameter 500- $\mu \mathrm{m}$-length CdSe NW. b-g, PL images of the CdSe NW locally excited at different positions along its length. h, Propagation-length-dependent PL output intensities of the CdSe NW at wavelength of 710,720 and $730 \mathrm{~nm}$, respectively. The corresponding curves of the experimental data are fitted using a first-order exponential decay. 


\section{Coupling efficiency between a CdSe NW and a Ag NW}

To estimate the coupling efficiency between the CdSe NW and the Ag NW in the coupled structure (Figure S4a), we excite the CdSe NW at the right side of the coupling area using a 532-nm light, and image the outputs of the NWs using a calibrated CCD camera (DXM1200F, Nikon) in dark-field mode without saturation (Figure S4b). By selecting an $60 \times 60$ pixel area (centered on the output spot) of the captured image, transforming the brightness into gray level information (a similar approach used in previous work ${ }^{2}$ ), and summing up the grey values of the corresponding area of each NW, we obtain the fractional output from the Ag NW of about $15 \%$ (the ratio of output intensity of the Ag NW to the total output of the both NWs). With the propagation loss of the Ag NW (for a 7.5- $\mu \mathrm{m}$ propagating length from coupling point to the end-facet) of about $3 \mathrm{~dB}(0.43 \mathrm{~dB} / \mu \mathrm{m})$ [Ref.2], the propagating loss of the CdSe NW (from the coupling region to the CdSe end-facet) of about $3 \mathrm{~dB}$ (about $224 \mathrm{~cm}^{-1}$ ) [Ref.3], and the end-facet reflectivity of $25 \%$ for the $\mathrm{Ag} \mathrm{NW}$ and $1 \%$ [Ref.4] for the CdSe NW, we determine a photon-to-plasmon coupling efficiency of $20 \%$.

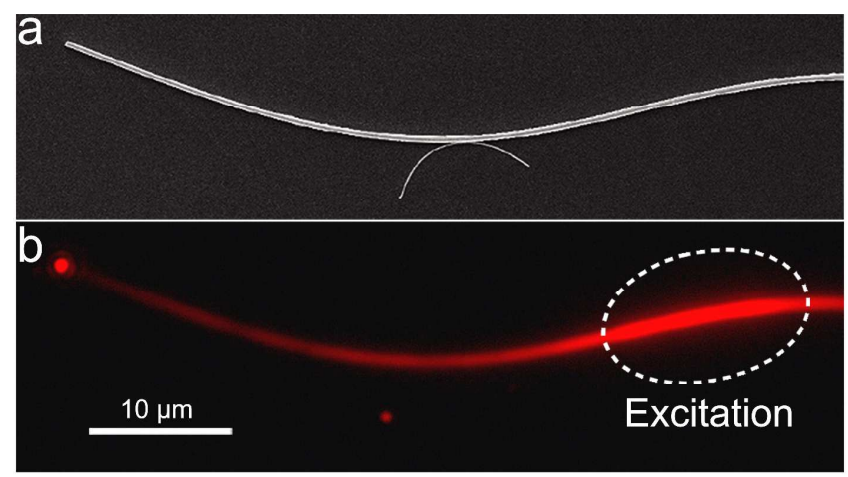

Figure S4. Coupling efficiency estimation. a, A SEM image of the coupled hybrid cavity composed of the CdSe and Ag NW. The left segment of CdSe NW and the left segment of Ag NW is 28.6 and $7.5 \mu \mathrm{m}$ in length, respectively. $\mathbf{b}$, Microscope image of the Y-shape coupler when exciting on the right segment of CdSe NW. 


\section{Calculation of FSR of the hybrid cavity}

Theoretically, FSR of the hybrid cavity can be estimated by

$F S R=\lambda^{2} /\left(2 \times\left(L_{C d S e} n_{g, C d S e}+L_{A g} n_{g, A g}\right)\right)$,

where $\lambda=723 \mathrm{~nm}, L_{\text {CdSe }}=28.6 \mu \mathrm{m}, L_{A g}=3.7 \mu \mathrm{m}$.

To obtain the group indices ( $n_{g, C d S e}$ and $n_{g, A g}$ ), we excite a 8 - $\mu$ m-length CdSe NW (cut from the same CdSe NW used here) to lasing using 532-nm laser pulses, acquire an FSR of $5.8 \mathrm{~nm}$ around $722.5 \mathrm{~nm}$ from the lasing spectrum, and obtain the group index $n_{g, \text { case }} \approx 5.6$. Similarly, for the $\mathrm{Ag} \mathrm{NW}$, a group index of $1.8\left(n_{g, A g} \approx 1.8\right)$ is obtained from Figure S2.b.

With above-mentioned parameters, we finally obtained a calculated FSR of $1.54 \mathrm{~nm}$ for the hybrid cavity, which agrees well with the experimental measurement $(1.50 \mathrm{~nm})$. The slight difference may be caused by the negligence of coupling length in the calculation process. 
Micromanipulation process of assembling the hybrid NW cavity
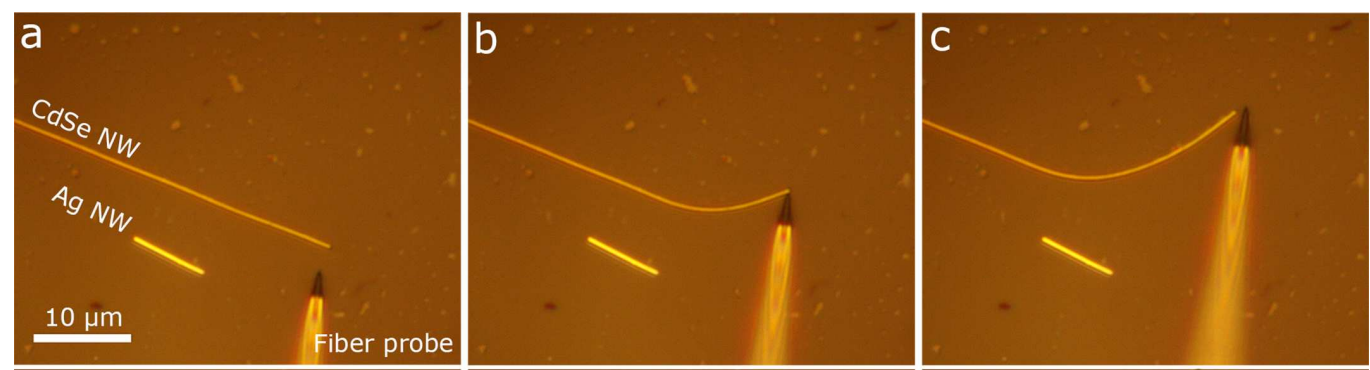

d
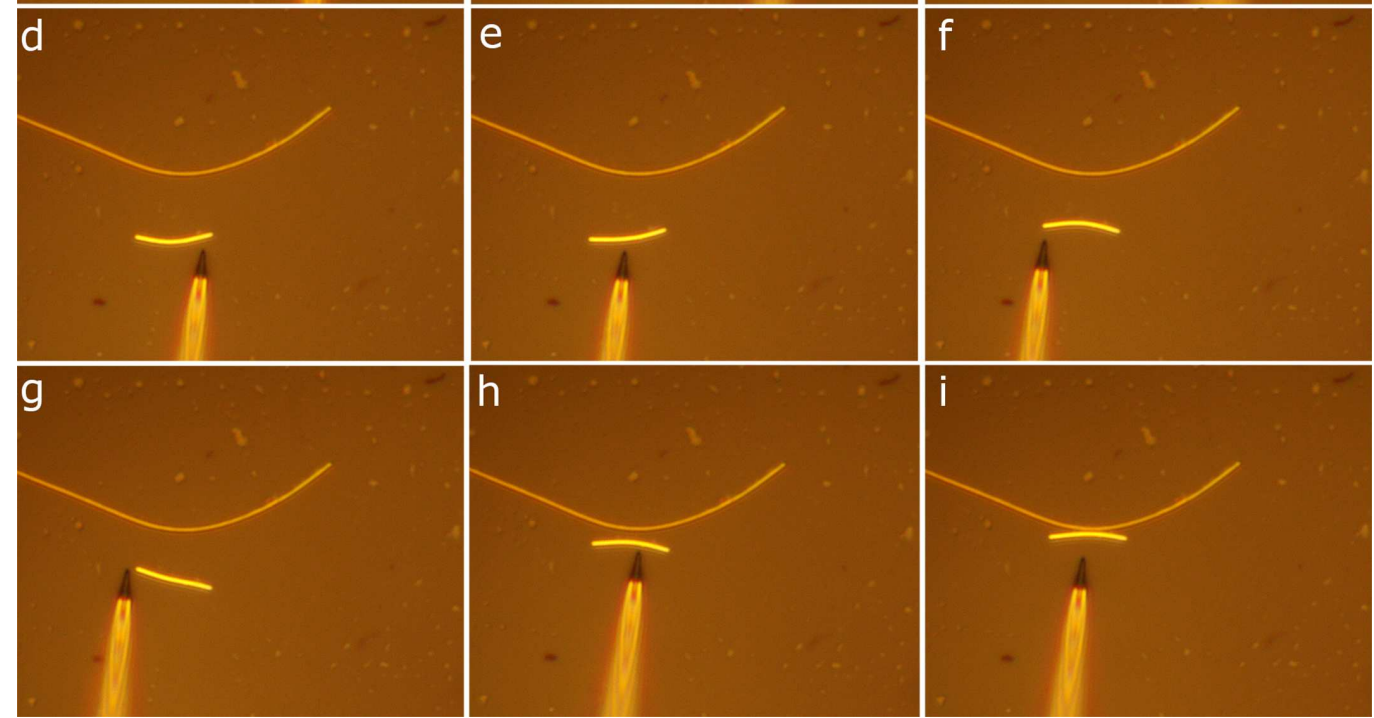

Figure S5. Micromanipulation process of assembling the hybrid NW cavity. a, The micromanipulation was carried out by a homemade fiber probe mounted on a precisely controlled 3-dimension moving stage under an optical microscope equipped with a super-long-distance objective. A Ag NW and a CdSe NW are deposited on the surface of a $\mathrm{MgF}_{2}$ substrate, and placed in parallel via micromanipulation with the fiber probe. b-c, The CdSe NW is bent to a v-shape profile using the fiber probe. $\mathbf{d}$-h, The Ag NW is pushed toward the bending area of the CdSe NW using the fiber probe. i, When the Ag NW is finally brought into contact with the CdSe NW, the hybrid NW cavity is assembled. 


\section{Mode area estimation of the Ag NW}

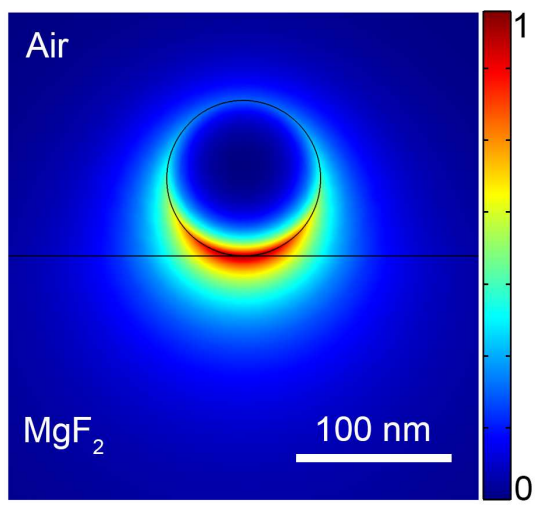

Figure S6. Mode area estimation of the Ag NW. Energy density distribution on the cross section of a 100-nm-diameter Ag NW placed on the $\mathrm{MgF}_{2}$ substrate. The wavelength used here is $723 \mathrm{~nm}$, corresponding to the dominant lasing peak in Figure 3. The mode area is calculated as $0.004 \mu \mathrm{m}^{2}$ [Ref.5].

\section{References}

(1) Ditlbacher, H. et al. Silver nanowires as surface plasmon resonators. Phys. Rev. Lett. 95, 257403 (2005).

(2) Ma, Y. et al. Direct measurement of propagation losses in silver nanowires. Opt. Lett. 35, 1160-1162 (2010).

(3) Xiao, Y. et al. Single-nanowire single-mode laser. Nano Lett. 11, 1122-1126 (2011).

(4) Wang, S. et al. Endface reflectivities of optical nanowires. Opt. Express 17, 10881-10886 (2009).

(5) Wang, Y., Ma, Y., Guo, X., Tong, L. Single-mode plasmonic waveguiding properties of metal nanowires with dielectric substrates. Opt. Express 20, 19006-19015 (2012). 